\title{
Smile detection using hybrid face representation
}

\begin{abstract}
Smile detection has attracted considerable amount of research interests in the domain of computer vision. It possesses several potential applications in gaming, human-to-computer and human-to-human interaction. This paper investigates the challenging problem of smile detection from face images acquired under unconstrained conditions. First, a locally weighted multiblock shape-texture descriptor is proposed to extract detailed local and global information from faces with diverse variations such as orientation, illumination, pose, and occlusion. The proposed technique combines the robustness of pyramid histogram of oriented gradient and local binary pattern for image feature representation using an adaptive local weight assignment. By locally weighting the descriptors from very dense patches of the image, we induce discriminating local spatial context to the distribution of the descriptions from the face image. Second, in order to minimize redundancy and extract the most relevant facial information from the feature vectors, a correlation based filter feature selection approach is adopted. Finally, kernel based classifiers such as support vector machine and kernel extreme learning machine are utilized for performing classification. Based on our findings, the proposed framework provides very competitive detection rate to related approaches that have exploited image alignment as an important stage for improving performance of smile detection.
\end{abstract}

Keyword: Smile detection; Unconstrained face images; Local feature descriptors; Feature selection;Kernel extreme learning machine; Support vector machine 